\title{
Dynamical decoupling of electron spins in phosphorus-doped silicon
}

\author{
RONG Xing, WANG Ya, YANG JiaHui, ZHU JinXian, XU WanJie, FENG PengBo, \\ WEN XuJie, SU JiHu \& DU JiangFeng"

\begin{abstract}
HeFei National Laboratory for Physical Sciences at Microscale and Department of Modern Physics, University of Science and Technology of
\end{abstract} \\ China, HeFei 230026, China
}

Received October 9, 2010; accepted November 2, 2010

\begin{abstract}
Quantum coherence is an important enabling feature underpinning quantum computation. However, because of couplings with its noisy surrounding environment, qubits suffer from the decoherence effects. The dynamical decoupling (DD) technique uses pulse-induced qubit flips to effectively mitigate couplings between qubits and environment. Optimal DD eliminates dephasing up to a given order with the minimum number of pulses. In this paper, we first introduce our recent work on prolonging electron spin coherence in $\gamma$-irradiated malonic acid crystals and analyze different decoherence mechanisms in this solid system. Then we focus on electron spin relaxation properties in another system, phosphorous-doped silicon ( $\mathrm{Si}: \mathrm{P}$ ) crystals. These properties have been investigated by pulse electron paramagnetic resonance (EPR). We also investigate the performance of the dynamical decoupling technique on this system. Using 8-pulse periodic DD, the coherence time can be extended to $296 \mu$ s compared with $112 \mu$ s with one-pulse control.
\end{abstract}

decoherence, dynamical decoupling, quantum computation

Citation: $\quad$ Rong X, Wang Y, Yang J H, et al. Dynamical decoupling of electron spins in phosphorus-doped silicon. Chinese Sci Bull, 2011, 56: 591-597, doi: 10.1007/s11434-010-4321-y

Decoherence, i.e. loss of coherence, is one of the main obstacles in implementing large-scale quantum computation [1]. Various strategies have been proposed to suppress decoherence, including quantum error-correction [2-4], decoherence free subspace [5-7] and dynamical decoupling [8-20]. Dynamical decoupling (DD) can be traced to the spin-echo technique [21] in NMR. It uses stroboscopic qubit flips to modify system-environment interaction so that the effect is close to self-canceling. The periodic Carr-PurcellMeiboom-Gill sequence [22] employs a series of refocusing pulses to dynamically decouple the system from its noisy environment. Concentrated dynamical decoupling [10] can suppress decoherence up to arbitrary order by an exponentially increasing number of pulses. Because errors are inevitably introduced by the control pulses, it is desirable to minimize their number used to realize dynamical decoupling to within some given level of precision. Optimal DD

*Corresponding author (email: djf@ustc.edu.cn) sequence was discovered by Uhrig to suppress pure dephasing of a single qubit coupled to a boson bath with a hard frequency cutoff [14]. This Uhrig dynamical decoupling (UDD) was later conjectured [15] and rigorously proved to be model-independent [16]. The first experimental study of UDD was conducted using trapped ions with ambient noise mimicked by modulating the controlling system [17]. In our recent experiment [23], we demonstrated extended coherence of electron spins in a solid system, specifically, radial electron spins in $\gamma$-irradiated malonic acid crystals, with UDD from $50 \mathrm{~K}$ through to room temperature. This optimal dynamical decoupling can also be applied to other solid-state systems, such as diamond with nitrogenvacancy centers which have also been realized in recent works [18-20]. In this paper, we report on the investigations into relaxation properties of phosphorous-doped silicon crystals lauded as an attractive candidate for spin-based quantum computing. We also investigated the performance of periodic dynamical decoupling (PDD) in this system. The 
coherence time of the electron spin has been prolonged to $296 \mu$ s with 8-pulse PDD control from $112 \mu$ s with onepulse control.

The main idea of the dynamical decoupling technique is to eliminate the coupling between qubit and its noisy environment. Taking an electron spin as a qubit, the Hamiltonian which includes the qubit, the environment and the interaction between the qubit and its environment can be expressed as

$$
H=\gamma_{e}\left(\vec{B}_{0}+\delta \vec{B}\right) \cdot \vec{\sigma} / 2+H_{E},
$$

where $\gamma_{e}$ is the electron gyromagnetic ratio, $\vec{B}_{0}$ is the external magnetic field, and $\delta \vec{B}$ is the fluctuation of the local magnetic field due to the surrounding environmental spin bath. The Pauli matrices $\vec{\sigma}=\left(\sigma_{x}, \sigma_{y}, \sigma_{z}\right)$ represent the quantized spin angular momentum of electron, $H_{E}$ contains the many-body interaction within the environment, while $\delta \vec{B}$ contains a static fluctuation resulting from thermal distribution of environmental states, usually referred to as inhomogeneous broadening.

DD uses repetitive $\pi$-pulses to flip the spin qubits so that the random magnetic field felt by the qubits before and after the pulses cancel each other. UDD can suppress decoherence up to order $N$ with a minimum number $(N)$ of pulses. In the pure dephasing model, where the random field is along a fixed axis, say the $z$ axis, the instants $t_{j}(j \in$ $\{1,2, \cdots, N\})$ at which the $N \pi$-pulses are applied along a perpendicular axis are given by

$$
t_{j}=T \sin ^{2} \frac{j \pi}{2(N+1)},
$$

where $T$ is the period of the pulse sequence. For PDD the instants are $t_{j}=T(2 j-1) /(2 N)$. Hereafter, we abbreviate $N$-pulse UDD and PDD to UDDN and PDDN, respectively. It has recently been proved that regardless of the interaction model between qubits and bath, UDD can preserve coherence up to order $O\left(T^{N+1}\right)$ [16]. The pulse sequences UDD1-UDD7 are shown in Figure 1(c) as well as the PDD7 pulse sequence. The first $\pi / 2$ pulse generates coherence while the following $N \pi$-pulses essentially preserve this coherence. An echo with area corresponding to the intensity of the coherence forms at time $t$. The decay of the echo signal with time $t$ has been recorded and analyzed under different DD pulse controls.

\section{Experiment}

\subsection{Dynamical decoupling in $\gamma$-irradiated malonic acid crystals}

We present recent work on prolonging electron spin coherence in $\gamma$-irradiated malonic acid crystals by DD pulse se- quences [23]. A unit cell of this single crystal is shown in Figure 1(a). A hydrogen atom may be removed from the methylene $\left(\mathrm{CH}_{2}\right)$ group by $\gamma$-irradiation and a radical $(-\mathrm{CH})$ may be created. To eliminate other kinds of radicals that are present, irradiated crystals are kept in an oven at $67^{\circ} \mathrm{C}$ for about $15 \mathrm{~h}$. The spin-1/2 of the unpaired carbon valence electron is the object under consideration which is referred to as a qubit. The corresponding energy level diagram of this system is shown in Figure 1(b); the continuous wave (CW) spectrum is shown in Figure 1(d). The resonance peak is split because of hyperfine coupling to the spin of the $\alpha$-proton [24], i.e. the hydrogen nucleus in the radical. The interaction can be described by

$$
H_{\mathrm{C}-\mathrm{H}} \approx 2 \pi\left(A_{0}^{z z} I_{0}^{z}+A_{0}^{z x} I_{0}^{x}\right) \sigma_{z} / 2,
$$

where the coupling constants are $A_{0}^{z z}=-45 \mathrm{MHz}$ and $A_{0}^{z x}=26$ $\mathrm{MHz}$ with respect to the specified direction of the external field. Samples with three different concentrations were prepared. The number of electron spins contributing to the absorption line is proportional to the area of the absorption peak. The concentration of radical electron spins in the crystal is therefore determined by a comparison of the spectrum to that of a standard sample (TEMPO), under the same experimental conditions. The concentrations measured were $0.6,8$ and $32 \mathrm{ppm}$. To obtain different concentrations of radicals, the samples were prepared by irradiated at various dose rates and for various durations of time. For example, the $0.6 \mathrm{ppm}$ sample was irradiated at a dose rate of about 2 $\mathrm{Gy} / \mathrm{min}$ for $15 \mathrm{~min}$.

We address the ensembles of electron spins for the enhancement of the signal. In such ensembles, we can also investigate the decoherence caused by couplings between qubits that continues to be a pertinent issue in large-scale quantum computation. At the beginning of the experiment, a $\pi / 2$-pulse about the $x$-axis was applied to the ensemble to generate coherence. Next, the $N$ spin-flip pulses in either UDD or PDD sequences (see Figure 1(c)) were applied to prolonging the electron spin coherence. At time $t$, a refocused echo will be generated. Phase cycling was applied to avoiding the unwanted echoes in the multi-pulse experiments. The coherence decay profile showed an initial exponential decay followed by a rapid super-exponential drop. We calculated the decay lifetime $T_{2}$ for the signal to drop $1 / \mathrm{e}$ of its initial value; this was obtained by extrapolation using exponential fitting to the initial decay data. The experimental results for various concentrations of radicals at various temperatures are shown in Figures 2 and 3 along with theoretical calculations. Up to seven controlling pulses (in both UDD and PDD pulse sequences) were applied. The coherence time was prolonged with increasing pulse number, verifying the persistence of spin coherence via DD sequences. Comparison between Figure 2(a) and (b) verifies that UDD outperforms PDD sequences in this system. 
(a)

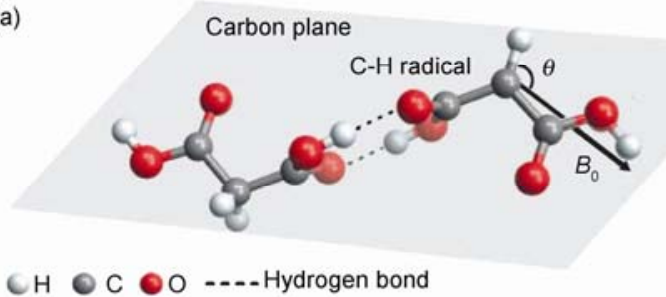

(c)

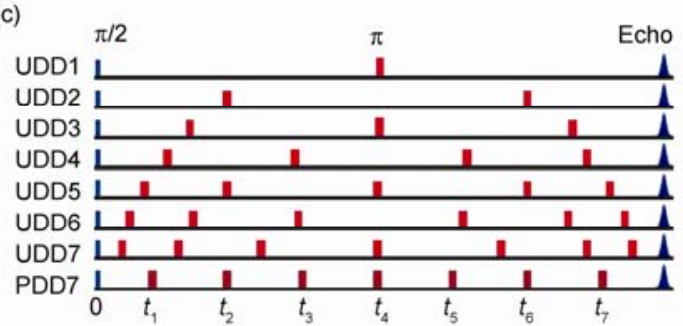

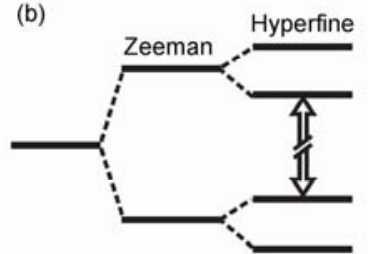

(d)

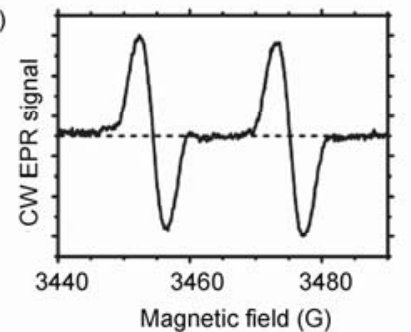

Figure 1 System and methods for the dynamical decoupling experiments [23]. (a) A unit cell of $\gamma$-irradiated malonic acid crystal; (b) energy level diagram of the coupled radical-proton system; (c) pulse sequences of UDD1-UDD7 and PDD7; (d) CW EPR spectrum of the radical was measured at 9.722 GHz.

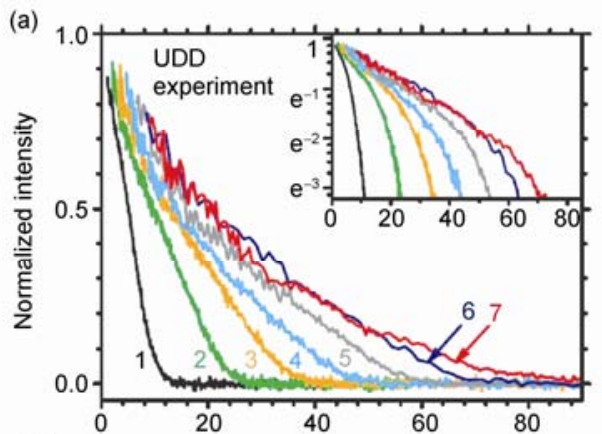

(c)

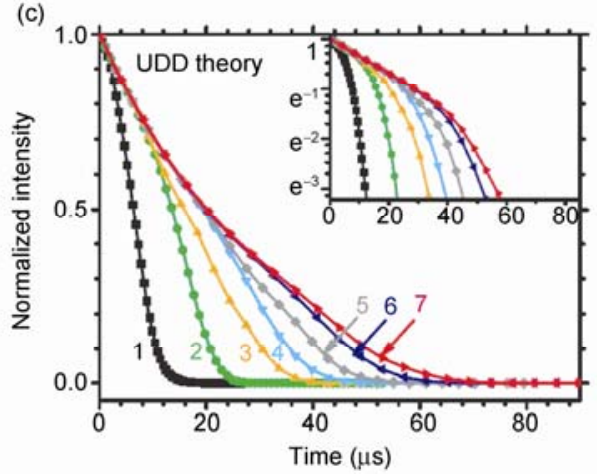

(b)

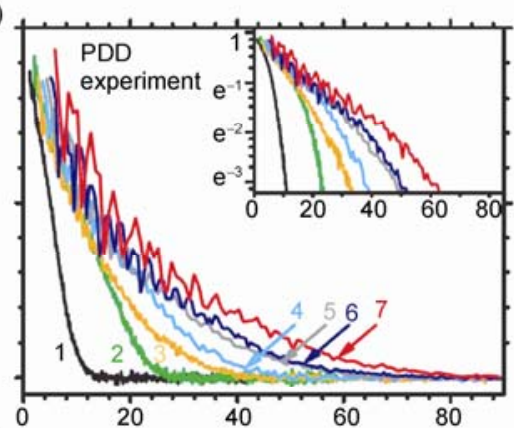

(d)

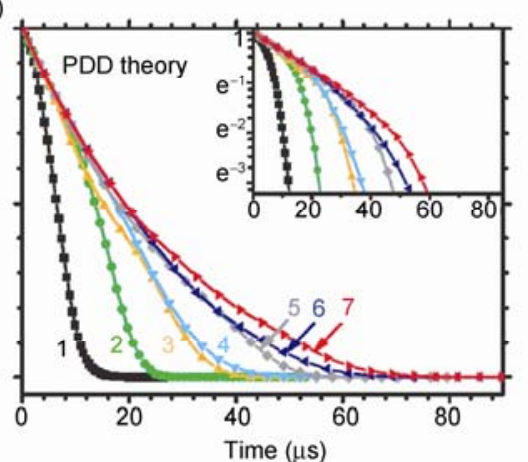

Figure 2 Preservation of electron spin coherence in malonic acid under UDD and PDD control [23]. (a) and (b), Coherence measured with respect to time; the experiments were carried out with the $0.6 \mathrm{ppm}$ sample at $50 \mathrm{~K}$ with various UDD and PDD pulse sequences; (c) and (d), the theoretical calculations corresponding to (a) and (b), respectively.

\subsection{Decoherence mechanisms in malonic acid single crystals}

The following mechanisms are considered relevant to decoherence in electron spin systems in solids: (1) direct relaxation due to spontaneous emission of photons; (2) spin-lattice relaxation via phonon scattering and spin-orbit interaction; (3) hyperfine interaction with nuclear spins; (4) coupling between qubits; and (5) coupling to other impurity electron spins. By comparing our experimental results with theoretical calculations, we can obtain detailed analysis of all of these decoherence mechanisms in malonic acid single crystals.

We can estimate the rate of spontaneous emission of photons at high temperature by the Fermi Golden rule: 


$$
\Gamma_{s}=2 \pi\left(\frac{k_{B} T}{\hbar \Omega}\right)\left(\frac{\hbar \Omega \gamma_{e}^{2} / 4}{c^{2} \varepsilon_{0}}\right)\left(\frac{2 \Omega^{2}}{3 \pi^{2} c^{3}}\right),
$$

where $\Omega \equiv \gamma_{e} B_{0}$ is the electron resonance frequency of about $10 \mathrm{GHz}$. Even if we consider the enhancement effect of the cavity, which has a $Q$ factor of about 100 , the spin relaxation rate due to direct photon emission is below $10^{-4} \mathrm{~s}^{-1}$ at room temperature and can be neglected.

Experiments at various temperatures (Figure 3(a)) singled out the effect of spin-lattice relaxation. Figure 3(a) displays the signals measured at 50, 100, 200 and $300 \mathrm{~K}$ after applying UDD5 on a single crystal sample of $\gamma$-irradiated malonic acid with concentration of $32 \mathrm{ppm}$. In our own experiments, thermal activation [25] was not resolved within decoherence-time. These small phonon scattering effects may be because of weak spin-orbit coupling in the carbon atoms. Thus, our experiments, performed at $50 \mathrm{~K}$ with $0.6 \mathrm{ppm}$ radical concentration, would be similar if measured at room temperature. Cooling the single crystals was solely in aide to obtain strong signal intensity.

Hyperfine interactions with nuclear spins generate a random local magnetic field (the Overhauser field):

$$
\Delta B_{\mathrm{hf}}=\langle\delta \hat{B} \delta \hat{B}\rangle^{1 / 2}=\gamma_{e}^{-1} 2 \pi \sqrt{\sum_{i=1}^{N}\left(A_{i}^{z z}\right)^{2}\left\langle I_{i}^{z} I_{i}^{z}\right\rangle},
$$

which is approximately $1.5 \mathrm{G}$. This is in agreement with that derived from the resonance line width $\Delta B_{\mathrm{hf}} \approx 2.0 \mathrm{G}$ in Figure $1(\mathrm{~d})$. The free induction decay (FID) time of the electron spin is estimated as $T_{2}{ }^{*}=40 \mathrm{~ns}$. This inhomogeneous broadening can be fully eliminated by the echoes.

More relevant is the dynamical fluctuation of the local Overhauser field generated by pairwise flip-flops of nuclear spins. The DD pulses applied in our experiment were mainly used to combat this decoherence mechanism; we verified that this scheme performed fairly well. In numerical simulations based on the microscopic theory developed to model such processes [26], we included up to four spin clusters and 500 nuclear spins around a single radical.

The dynamical Overhauser field fluctuation can also be caused by random nuclear displacements relative to the electron spin, especially by nuclei near the radical. For example, the $\alpha$-proton can hop between two locations where the $\mathrm{C}-\mathrm{H}$ bond of the radical is tilted by $\pm 12^{\circ}$ away from the carbon plane. An accurate understanding of the dynamics of the conformation requires detailed knowledge of electron states and hopping process that are not available at present. However, we can estimate the decoherence caused by such a mechanism by introducing a phenomenological decay factor $\exp \left(-T / T_{2}{ }^{\prime}\right)$, where $T_{2}{ }^{\prime}=30 \mu \mathrm{s}$ in all experiments.

Coupling between electron spin qubits and other radical electron spins can also result in a random local field. The static inhomogeneous broadening field $\delta B_{e e}$ in our samples ranged from 0.001 to $0.05 \mathrm{G}$, which is far less than the Overhauser field broadening. This is not important in the CW EPR spectrum and FID. However, electron spins are all near resonance and are flipped together by the $\pi$-pulses. DD sequences cannot fully eliminate such interactions and $\delta B_{e e}$
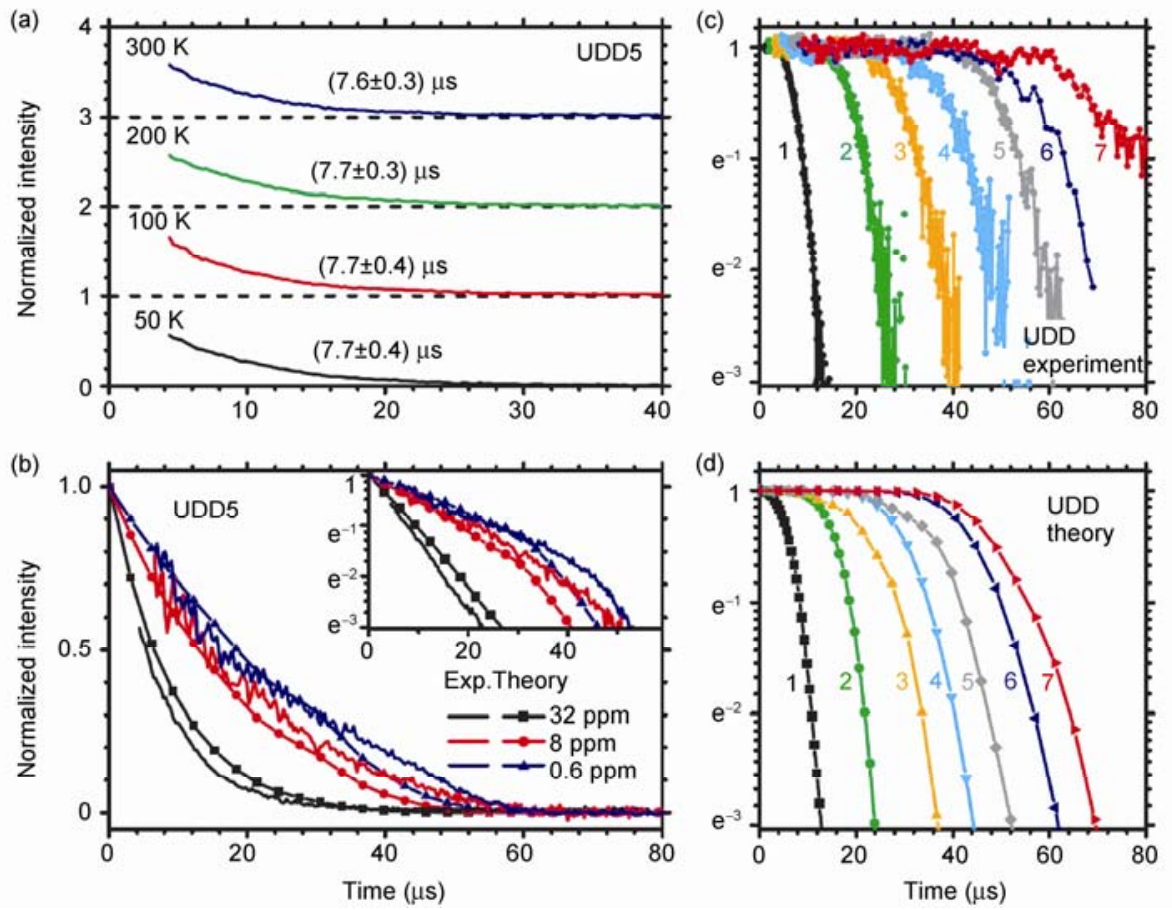

Figure 3 Effects of various decoherence mechanisms in malonic acid crystals [23]. (a) UDD5 echo signals of a 32 sample measured at 50, 100, 200, 300 K; (b) coherence time under UDD5 with concentrations of $0.6,8$ and $32 \mathrm{ppm}$ at $50 \mathrm{~K}$; (c) UDD1-UDD7 signals of a 0.06 ppm sample at $50 \mathrm{~K}$ with the initial exponential fit subtracted; (d) theoretical calculations for (c). 
will become important in echo signals. Thus, Figure 3(b) shows that after application of an UDD5 sequence, $T_{2}$ of the $32 \mathrm{ppm}$ sample is much shorter than that for the $0.06 \mathrm{ppm}$ sample, indicating that in high concentration samples, interactions between electron spins dominate the decoherence mechanism.

In Figure 3(c) and (d), we see that if we consider only the dynamical fluctuation of the Overhauser field caused by pairwise flipping of nuclear spins, which are dominated by nuclear dipole interactions, we can maintain electron spin coherence for a rather long time. Over longer periods, decoherence is dominated by other mechanisms arising from electron-electron interactions and conformation-hopping; this can explain the overall non-exponential feature in the coherence decay.

\subsection{Dynamical decoupling in phosphorous-doped sili- con (Si:P)}

Since Kane's original proposal [27], Si:P single crystals have become a promising spin qubit system for its long coherence times [28], the possibility of single-spin detection by electrical measurements [29-32], and mature material and fabrication technologies. The basic unit of the qubit ensemble in Si:P crystals consists of an unpaired electron spin along with a nuclear spin of ${ }^{31} \mathrm{P}$. At low temperatures, the electron is bound to the phosphorus nucleus and there is an isotropic hyperfine coupling [33] between the electron and nuclear spin. The system's Hamiltonian can be expressed as

$$
H_{0}=\omega_{S} S_{z}+\omega_{I} I_{z}+A \overrightarrow{\mathrm{S}} \cdot \vec{I},
$$

where the coupling constant is $A=117 \mathrm{MHz}$, and $\omega_{S}$ and $\omega_{I}$ denote Larmor frequencies of the electron and nuclear spins, respectively.

A sample of phosphorous-doped bulk silicon single crystals with an electron spin concentration of about $10^{16}$ spins $/ \mathrm{cm}^{-3}$ was also used. The sample was cooled down to $10 \mathrm{~K}$ with liquid helium. All data were recorded by a commercial pulsed EPR spectrometer (E580, Bruker). The FSED spectrum of this sample is reproduced in Figure 4(b). The splitting between the two peaks is caused by hyperfine coupling between the electron spin and the ${ }^{31} \mathrm{P}$ nuclear spin. The linewidth of the peaks is $2.24 \mathrm{G}$ indicating about $36 \mathrm{~ns}$ FID time for the electron spin. This inhomogeneous broadening can be eliminated by Hahn echo pulse. Figure 4(d) shows the Hahn echo decay of Si:P at $10 \mathrm{~K}$. The $T_{2}$ time, derived from the signal lifetime, was estimated to be 112 $\mu \mathrm{s}$. The dependence of the spin-lattice time $\left(T_{1}\right)$ on temperature has also been plotted in Figure 4(c). $T_{1}$ increases significantly as the sample is cooled. This dependence plotted in Arrhenius coordinates $\left(\log \left(1 / T_{1}\right)\right.$ vs. $\left.1 / T\right)$ is linear indicating that an Orbach mechanism dominates the relaxation (a)

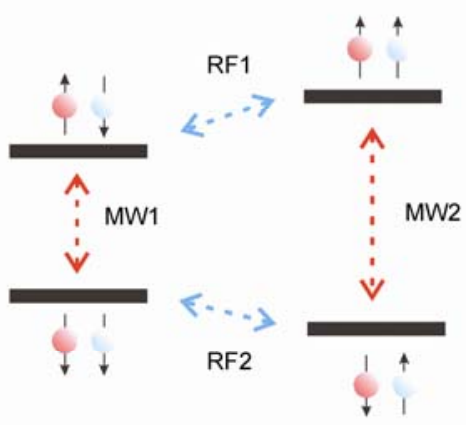

(c)

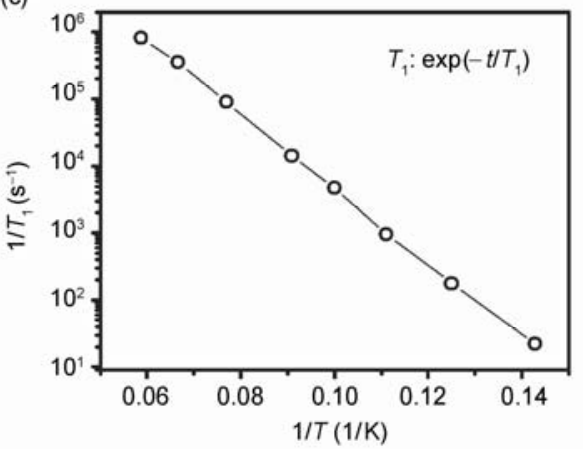

(b)
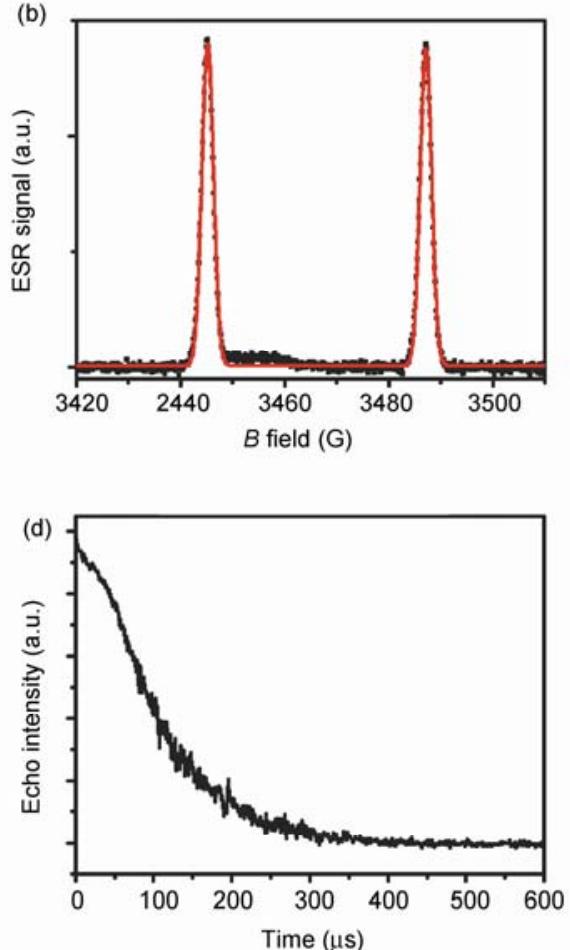

Figure 4 (a) The energy level diagram of a phosphorous-doped silicon single crystal. The red (blue) circles with an up (down) arrow label denotes the electron (nuclear) spin state $\left|M_{S}>\left(\mid M_{l}>\right)=\right| 0>(\mid 1>)$ ). There are two allowed electron (nuclear) spin transitions which are labeled as MW1 and MW2 (RF1 and RF2). (b) The field sweep echo detection (FSED) spectrum of Si:P at $10 \mathrm{~K}$. The experimental data are fitted by Gaussian line-shapes (red line); line-widths are about 2.24 G. (c) The spin-lattice times $\left(T_{1}\right)$ of Si:P dependency of temperature are plotted. (d) The Hahn echo decay has been obtained by a $\pi / 2-\tau-\pi-\tau$-echo pulse sequence. 
process at these temperatures [34]. Our result is similar with a previous experimental observation [35].

PDD pulse sequencing has been applied to testing the coherence persistence of the unpaired electron spin coherence at $10 \mathrm{~K}$. The results of our experiments are shown in Figure 5. Longer coherence times are achieved as pulse numbers increase. With a PDD1 sequence (i.e. the Hahn echo pulse sequence), the coherence time is about $112 \mu \mathrm{s}$. This is extended to $296 \mu$ s with PDD8 which is about 8300 times the signal lifetime of $36 \mathrm{~ns}$ in the absence of pulse control and 2.64 times of $112 \mu$ s with one-pulse control. Saturation in the coherence time has been observed while increasing PDD pulse numbers. A similar saturation has also been observed in our previous work [23]. The electron-electron spin dipole-dipole coupling contributes to this saturation.

\section{Conclusion}

We have given a brief review of our recent work in which we discussed the performance of dynamical decoupling pulses on $\gamma$-irradiated malonic acid crystals. Several decoherence mechanisms have been discussed and analyzed in this solid system. We then investigated $\mathrm{Si}: \mathrm{P}$ crystals, which have different decoherence mechanisms. The times $T_{1}$ and $T_{2}$ were measured by pulse EPR spectrometer. We also performed the PDD pulse sequence on electron spins in $\mathrm{Si}: \mathrm{P}$ and found that it can effectively prolong coherence times. The concentration of the electron spins of our sample is about $10^{16}$ spins $/ \mathrm{cm}^{-3}$ and the saturation of the coherence time with increasing PDD pulse number may be because of spin dipole-dipole coupling of the electron spins. Systems with low electron spin concentration will be of more interest

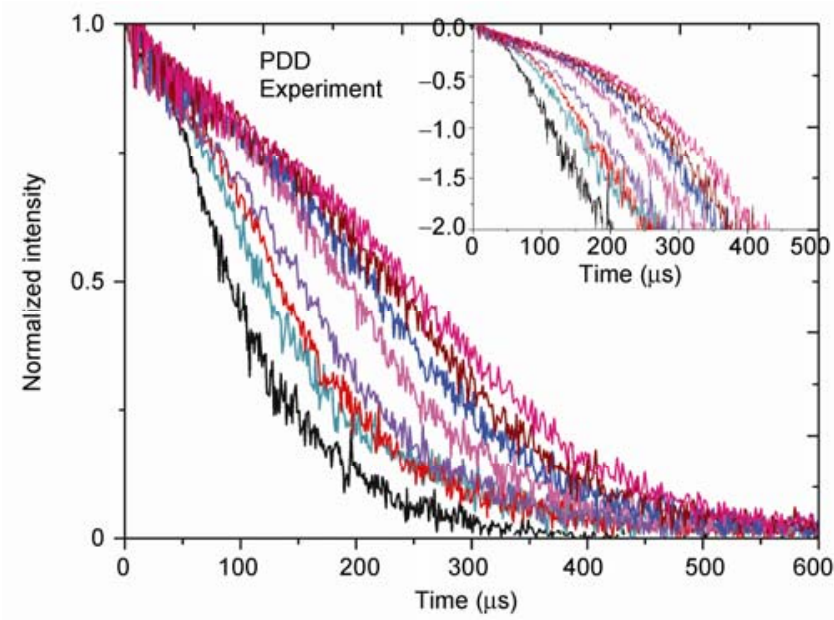

Figure 5 Electron spin echo decay under PDD control. The experimental data have been collected at $10 \mathrm{~K}$. Echo signal decays and the intensities have been normalized. Inset, semi-logarithmic plot of the same data; the variables plotted on the axes are the same as in the main panel. for further research. The UDD pulse sequencing is a good method to understand decoherence mechanisms of this solid system; thus, it is worthwhile performing UDD on this system in future research. The echo decay dependence on the angle between the external magnetic field and the single crystal [36] is also worthwhile investigating.

This work was supported by the National Natural Science Foundation of China, the Chinese Academy of Sciences, the Ministry of Education of PRC, the National Basic Research Program of China (2007CB925200).

1 Nielsen M A, Chuang I L. Quantum Computation and Quantum Information. Cambridge: Cambridge University Press , 2000

2 Shor P W. Scheme for reducing decoherence in quantum computer memory. Phys Rev A, 1995, 52: 2493-2496

3 Steane A M. Error correcting codes in quantum theory. Phys Rev Lett, 1996, 77: 793-797

4 Knill E. Quantum computing with realistically noisy devices. Nature, 2005, 434: 39-44

5 Duan L M, Guo G C. Preserving coherence in quantum computation by pairing quantum bits. Phys Rev Lett, 1997, 79: 1953-1956

6 Zanardi P, Rasetti M. Noiseless quantum codes. Phys Rev Lett, 1997 , 79: 3306-3309

7 Lidar D, Chuang I L, Whaley K B. Decoherence-free subspaces for quantum computation. Phys Rev Lett, 1998, 81: 2594-2597

8 Viola L, Knill E, Lloyd S. Dynamical decoupling of open quantum systems. Phys Rev Lett, 1999, 82: 2417-2421

9 Kern O, Alber G. Controlling quantum systems by embedded dynamical decoupling schemes. Phys Rev Lett, 2005, 95: 250501

10 Khodjasteh K, Lidar D A. Fault-tolerant quantum dynamical decoupling. Phys Rev Lett, 2005, 95: 180501

11 Santos L F, Viola L. Enhanced convergence and robust performance of randomized dynamical decoupling. Phys Rev Lett, 2006, 97: 150501

12 Yao W, Liu R B, Sham L J. Restoring coherence lost to a slow interacting mesoscopic spin bath. Phys Rev Lett, 2007, 98: 077602

13 Witzel W M, Das Sarma S. Concatenated dynamical decoupling in a solid-state spin bath. Phys Rev B, 2007, 76: 241303

14 Uhrig G S. Keeping a quantum bit alive by optimized $\pi$-pulse sequences. Phys Rev Lett, 2007, 98: 100504

15 Lee B, Witzel W M, Das Sarma S. Universal pulse sequence to minimize spin dephasing in the central spin decoherence problem. Phys Rev Lett, 2008, 100: 160505

16 Yang W, Liu R B. Universality of Uhrig dynamical decoupling for suppressing qubit pure dephasing and relaxation. Phys Rev Lett, 2008, 101: 180403

17 Biercuk M J, Uys H, VanDevender A P, et al. Optimized dynamical decoupling in a model quantum memory. Nature, 2009, 458: 996-1000

18 de Lange $\mathrm{G}$, Wang Z H, Ristè D, et al. Universal dynamical decoupling of a single solid-state spin from a spin bath. Science, 2010, 330: 60-63

19 Naydenov B, Dolde F, Hall L T, et al. Dynamical decoupling of a single electron spin at room temperature. arXiv: $1008.1953 \mathrm{v} 2$

20 Ryan C A, Hodges J S, Cory D G. Robust decoupling techniques to extend quantum coherence in diamond. Phys Rev Lett, 2010, 105: 200402

21 Hahn E. Spin echoes. Phys Rev, 1950, 80: 580-594

22 Schweiger A, Jeschke G. Principles of Pulse Electron Paramagnetic Resonance. Oxford: Oxford University Press, 2001

23 Du J F, Rong X, Zhao N, et al. Preserving electron spin coherence in solids by optimal dynamical decoupling. Nature, 2009, 461: 12651268

24 McConnell H M, Heller C, Cole T, et al. Radiation damage in organic crystals. I. $\mathrm{CH}(\mathrm{COOH})_{2}$ in malonic acid. J Am Chem Soc, 1960, 82: 766-775 
25 Dalton L R, Kwiram A L, Cowen J A. Electron spin-lattice and cross relaxation in irradiated malonic acid. Chem Phys Lett, 1972, 14: 77-81

26 Yang W, Liu R B. Quantum many-body theory of qubit decoherence in a finite size spin bath. Phys Rev B, 2008, 78: 085315

27 Kane B E. A silicon-based nuclear spin quantum computer. Nature, 1998, 393: 133-137

28 Tyryshkin A M, Lyon S A, Astashkin A V, et al. Electron spin relaxation times of phosphorus donors in silicon. Phys Rev B, 2003, 68: 193207

29 Stegner A R, Boehme C, Huebl H, et al. Electrical detection of coherent ${ }^{31} \mathrm{P}$ spin quantum states. Nat Phys, 2006, 2: 835-838

30 McCamey D R, Huebl H, Brandt M S et al. Electrically detected magnetic resonance in ion-implanted Si:P nanostructures. Appl Phys Lett, 2006, 89: 182115
31 Lo C C, Bokor J, Schenkel T, et al. Spin-dependent scattering off neutral antimony donors in Si-28 field-effect transistors. Appl Phys Lett, 2007, 91: 242106

32 Morello A, Pla J J, Zwanenburg F A. Single-shot readout of an electron spin in silicon. Nature, 2010, 467: 687-691

33 Feher G. Electron spin resonance experiments on donors in silicon I: Electronic structure of donors by the electron nuclear double resonance technique. Phys Rev, 1959, 114: 1219-1244

34 Orbach R. Spin-lattice relaxation in rare-earth salts. Proc Phys Soc Lond Ser A, 1961, 264: 458-484

35 Lyon S A. Relaxation of candidate electron spin qubits. In: Smulko J M, Blanter Y, Dykman M, et al, eds. Noise and Information in Nanoelectronics, Sensors, and Standards II. 2004, 5472: 97-106

36 Tyryshkin A M, Morton J J R, Benjamin S C, et al. Coherence of spin qubits in silicon. J Phys-Cond Matter, 2006, 18: S783

Open Access This article is distributed under the terms of the Creative Commons Attribution License which permits any use, distribution, and reproduction in any medium, provided the original author(s) and source are credited. 